\title{
Can Neuroscience Improve Addiction Treatment and Policies?
}

\author{
David Nutt, FMedSci, ${ }^{1}$ \\ A. Thomas McLellan, $\mathrm{PhD}^{2}$
}

\begin{abstract}
The main target of alcohol and other drug self-administration is the brain. For this reason understanding of brain mechanisms (i.e., neuroscience research) may offer important insights for creating effective prevention and treatment interventions, as well as fair and appropriate drug policies. Fair and appropriate drug policies (public laws and regulations) are expected to reduce the harms of drugs to the user and to society in general, while minimizing unintended but related policy "side effects."

In this paper we explore how more informed understanding of neuroscience may help to improve the effectiveness and reduce the unintended side effects of contemporary drug policies. The article goes on to suggest some rational ways neuroscience may better inform and assist in policy decisions. We cover currently illegal drugs plus others such as alcohol and tobacco and touch on the new and emerging substances-legal highs.
\end{abstract}

Key Words: Neuroscience, addiction treatment, drug policy, alcohol, tobacco.

Recommended Citation: Nutt D, McLellan AT. Can neuroscience improve addiction treatment and policies? Public Health Reviews. 2014;35: epub ahead of print.

\section{THE NEUROSCIENCE OF ADDICTION}

Neuroscience is the study of brain mechanisms - from genetic and molecular mechanisms to psychological processes and clinical conditions. Neuroscience is now one of the major disciplines in science, but this is a recent phenomenon with the blossoming of emerging findings resulting from

\footnotetext{
${ }^{1}$ Imperial College London, London, UK.

${ }^{2}$ Treatment Research Institute, Philadelphia, PA, USA.
}

Corresponding Author Contact Information: David Nutt at d.nutt@imperial.ac.uk; Imperial College London, Burlington Danes Building, Hammersmith Hospital, DuCane Rd., London W12 0NN, UK. 
molecular biology, genetics and brain imaging techniques such as positron emission tomography (PET) and functional magnetic resonance imaging (fMRI). These new tools are providing important new insights into models of brain disorders and the integrative structure of the brain, and a growing understanding on regional brain interactions and connectivity.

\section{Neurotransmitters and Receptors in Addiction}

Drugs are self-administered because they make people feel differentusually better-in terms of mood, alertness, wellbeing, etc. Most of the changes associated with drug use are immediate-often much faster than comparable changes produced by naturally occurring stimuli. The rapid action of most drugs of abuse on brain reward mechanisms likely accounts for much of their attraction. While many physiological activities such as exercise, sex and eating can release neurotransmitters such as dopamine and produce pleasure or rewarding experiences,${ }^{1}$ drugs of abuse tend to produce more rapid and in some cases very high levels of reward neurotransmitters such as dopamine. Research suggests this rapid release of reward-inducing experiences is a significant part of the transition from exploratory, to regular, to compulsive use (i.e., addiction). There has been a considerable interest in the role of dopamine in mediating the actions of drugs of abuse since animal studies first suggested this might be a final common pathway of rewarding drug effects. ${ }^{2,3}$ Detecting this in the human brain is now feasible using PET or single photon emission computer tomography (SPECT) imaging with dopamine sensitive tracers such as $11 \mathrm{C}$-raclopride. ${ }^{4}$

It is interesting that some sought-after drug effects are delayed or mixed with negative effects. For example, tobacco smokers usually have to develop tolerance to the unpleasantness of filling their mouth and lungs with smoke before getting the "benefits" of nicotine. Some psychedelic drugs (e.g., ayahuasca) can be acutely distressing during the "trip" but leave many users feeling much better afterwards, often for months or years. Finally, following regular use of many of the more potent drugs (e.g., alcohol, opioids, cocaine, etc.) there can be serious negative side effects such as withdrawal and craving following the cessation of the drug use. The complex interactions among the nature, sequence and duration of the positive effects and negative effects of the common drugs of abuse appear to account for their appeal and their "addictive" qualities.

\section{Neuro-circuitry and Genetic Expression in Addiction}

Knowledge of the dopamine-based reward circuit; how that circuit interacts with other inhibitory and motivational circuits; and how those circuits 
affect genetic expression of traits like compulsive use may help us make predictions about the abuse potential of new drugs. This is regardless of whether that drug is a naturally occurring compound found in plants (e.g., THC or nicotine), or whether it has been synthesized in a laboratorythe so-called "designer drugs." However, the acute release of rewardproducing neurotransmitters such as dopamine is just part of the overall picture of drug "addiction." We next need to understand how drug-induced changes in neurotransmitter release and metabolism gradually lead to changes in groups of neurons that comprise circuits within the brain; and in turn, how those circuits come to exert control of behavioral functions such as learning, memory, motivation and inhibition.

Neuroscience has not been necessary to identify addictive behaviors or to account for the spread of these behaviors, since traditional epidemiological research has been instructive in those important elements of addiction. However, the description and the prediction of the development of addictive behaviors among individuals and population subgroups are likely to be helped with greater neuroscience information. Through the study of neuroscience over the past several years we now know that the long-standing idea that a single neurotransmitter response (e.g., dopamine release) is responsible for all pleasurable drug actions is no longer viable..$^{5}$ It has become clear that alcohol and other drugs have multiple and different effects on brain functions. Stimulants act to release or increase the actions of dopamine. Opioids act to mimic the effects of the endogenous opioid neurotransmitters the endorphins. Alcohol works to block the major excitatory neurotransmitter glutamate and enhance the major inhibitory neurotransmitter GABA. Tobacco works largely to deliver nicotine to the brain and cannabis delivers psychoactive cannabinoids particularly THC and cannabidiol. Empathogens like MDMA act via the release of 5-HT noradrenaline and dopamine, and psychedelics act as agonists to stimulate 5-HT2A receptors. ${ }^{6}$ In turn, this level of basic biological understanding has helped us better grasp individual variation in initial response to drugs and in susceptibility to addiction. Moreover, at least in animal models, there is evidence that variables such as dopamine receptor number or function can be changed and this produces alterations of drug use and possibly addiction. ${ }^{7}$

However, these new tools of modern neuroscience have also shown that there are many more receptor systems than previously known; that these systems interact in different ways; that within each system the same receptors may be affected by agonists, partial agonists or antagonists; and that over time and with repeated use the same drug, acting upon the same receptors may produce very different neurochemical, experiential and behavioral effects. To make these already complicated interactions even more complex, 
we now know that these initial and protracted effects are jointly influenced by genetic, learned and environmental variables as drug "use" proceeds to "abuse" and ultimately "addiction." Many of these effects have been well described by Volkow et al. $2011^{8}$ in a recent review as the intersection of four brain pathways involving: memory (hippocampus and amygdala), reward (ventral striatum/n accumbens), drive/salience ((orbitofrontal cortex (OFC)) and cognitive control (dorso-lateral pre-frontal cortex (DLPFC)).

In simple terms addiction can be considered a gradually acquired (through repeated use) loss of the sophisticated neurochemical and reciprocal balance among these four regions such that drives become less well regulated by "top down" cognitive control from the DLPFC; concurrent with likely increased salience and greater impulsivity (through learned drive salience) and thence more drug use (Figure 1). We know this much from advances in neuroscience-but we still know very little about the genetic, environmental and learned factors that promote these changes. These are important limitations that have impeded our ability to translate existing neuroscience findings into more effective medications and behavioral interventions.

\section{Brain Circuitry of Addiction}

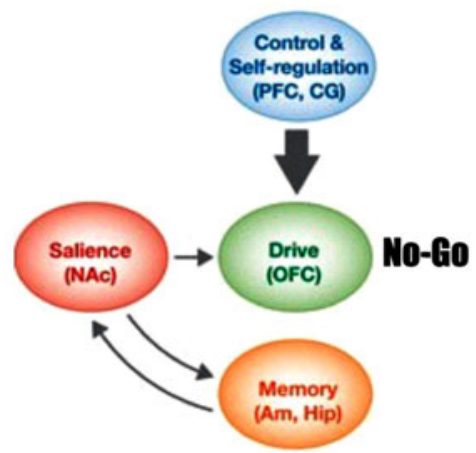

Non-addicted circuitry

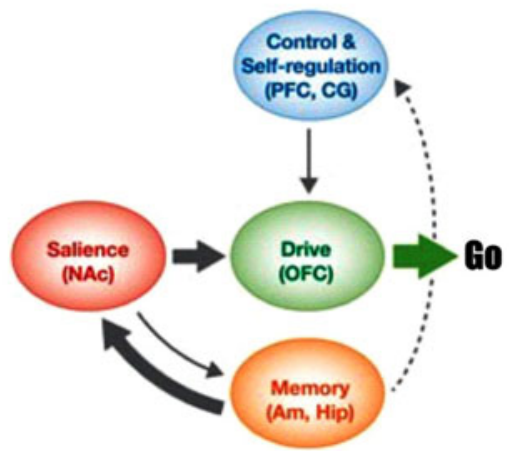

Addicted circuitry

Fig. 1. Brain Processes Underpinning Drug Use and Addiction. Model of addiction as a result of chronic substance use within independent and overlapping circuits of the brain. Compared with the non-addicted circuitry (left), the salience value of a drug (red) and its associated cues (orange) becomes exaggerated in the addicted circuitry (right). The strength of inhibitory control is weakened (blue), together with unrestrained motivation/drive (green). This results in compulsive substance use and recurrent relapse in addiction.

Source: Based on Volkow, et al. 2012.29 


\section{Neuroscience and Voluntary Control of Behavior}

It is important to note the role of voluntary and involuntary processes here-as public understanding and resulting policies have long been based upon the idea that alcohol and other drug addictions are unlike other "illnesses" because they are self-induced. In fact, drug addiction $i s$ at least partially self-induced-but so are many other acquired chronic illnesses such as type 2 diabetes, many forms of hypertension, asthma, and even chronic pain. The initial act of drinking alcohol, smoking marijuana or injecting heroin are completely voluntary, usually prompted by curiosity and/or social interactions, and even, in the case of alcohol and tobacco by sophisticated advertising.

But as we have learned from neuroscience research, most common drugs of abuse produce rapid neurochemical changes that can (but do not always) lead to powerful experiences, the triggering of learning and memory and likely the expression of dormant genes. ${ }^{9}$ In particular, there is emerging animal evidence as well as evidence from human twin studies, that genetic alterations in the density of dopamine and other neurotransmitter receptors can produce marked differences in the immediate and longer term effects of drugs of abuse. Put differently, while all substance use tends to begin in a voluntary manner, the still unexplained interactions between a particular drug of abuse, a specific genetic vulnerability, and a particular set of environmental circumstances appear to govern the eventual course of successive drug use and likely the incremental change from voluntary, controlled substance "use" to compulsive "addiction."

In this regard, animal studies of cocaine administration in rats have shown wide variability in response to that drug depending upon genetic variations in the dopamine D2 receptors. Rats with low levels of receptors tended to self administer more cocaine than rats with normal/high receptor levels. ${ }^{10}$ This finding fits with human data that the liking of stimulants by humans is correlated with dopamine receptor density-the more dopamine receptors in the brain, the less stimulants are liked. ${ }^{11}$ Changes in receptor function and neurotransmitter release have also been reported. The majority of imaging studies on dopamine receptors in stimulant and alcohol addicts find reduced dopamine DRD2 receptor number in the basal ganglia ${ }^{12}$ while unaffected family members have higher levels of these receptors. ${ }^{13}$

These reductions may reflect some adaptive or even toxic changes from drug use but may also reflect some underlying vulnerability. Thus, the repeated self-administration of most drugs of abuse can produce increasingly more complex and profound changes in multiple neurochemical circuits, brain region interactions and corresponding changes in memory, motivation 
and inhibitory control. ${ }^{9}$ The mechanisms underlying these memories are likely related to synaptic plasticity driven through the NMDA and other glutamate receptors. The power of these can overcome even the pharmacological tendency of some sedative drugs such as alcohol, ketamine and GHB to directly impair memory deposition. Thus, the level of voluntary selfcontrol may erode, as tolerance develops to the initially intensely positive effects of a drug, combined with parallel development of significant levels of withdrawal and craving.

It is also true that there can be significant brain injury and loss of function associated with use of drugs. We can say with some certainty that alcohol damages the brain in a significant proportion of those who use it chronically and heavily. These changes have been described at postmortem physical examinations for centuries, and more recently proven in the living human brain using imaging studies ranging from x-rays (with air contrast) to MRI. Indeed, the injurious effects of alcohol on the brain have confounded many of the claims of brain damage by other drugs that are used in conjunction with alcohol. ${ }^{14} \mathrm{~A}$ recent example showed how extreme use of some drugs, particularly when take via the intravenous route can lead to enduring brain damage..$^{15}$ This may be particularly true in the case of stimulants such as cocaine and metamfetamine that alter dopamine function and may also reduce pre-frontal cortex size and function.

\section{NEUROSCIENCE-BASED ADVANCES IN TREATMENT AND POLICY}

To date, drug policy has not been well served by findings from neuroscience. This is in part because most drug policies have been in existence for decades or longer, while much of what we have learned in neuroscience has come only recently with advances in brain imaging, molecular biology and genetic studies. Also, the information provided by contemporary neuroscience is quite complicated and understanding requires both information and integration that are difficult to implement through relatively blunt, simplistic policies and regulations.

An obvious example of the gap between policy and science in this area is the designation of what constitutes a legal and an illegal drug. This has been well described in a systematic analysis of twenty drugs using multicriteria decision analysis incorporating 16 parameters of harm, many of which are related to brain mechanisms. ${ }^{16}$ The results from this analysis provoke a startling lack of correspondence between the (now) known public harms associated with drugs and the level of prohibition placed upon them. 
Easily the best example is alcohol, which is almost certainly the most damaging drug to the brain, but yet legal and widely commercialized in most countries. In contrast, cannabis produces relatively little brain damage and yet is illegal in most countries. ${ }^{17}$

Similar science-policy anomalies exist between a drug's potential to produce addiction and the level of social policy control placed upon the drug. For example, many closely controlled drugs do not reliably produce compulsive, uncontrolled use and yet are widely sanctioned publicly. Perhaps the best example here is psychedelics. In contrast the most addictive drug_at least as defined by the proportion of those who are addicted relative to those who have tried the drug - is tobacco, ${ }^{18}$ which is legal and commercialized in almost all countries (the exception being Bhutan).

To provide still more complexity, many of the "drugs of abuse" also have significant medicinal value (e.g., opioids, benzodiazepines, and several of the constituents of tobacco and marijuana plants). Although opioids and amphetamines are controlled they are also necessary medicines (for pain and attention deficit disorder, respectively) so arrangements are made in most countries for them to be made available for prescription use. Cannabis has for many thousands of years been used as a treatment for a range of pain and stress disorders yet was banned by the 1961 United Nations convention from such uses; although now in many states in the United States and some other countries (e.g., Uruguay and The Netherlands) this limitation has/is being overturned.

Other drugs with lesser harms also have therapeutic uses but are not currently available for treatments. These include psychedelics, which have been used to successfully treat alcoholism with an effect size equal or better than that of current treatments such as acamprosate. ${ }^{19}$ Another psychedelic, psilocybin has been shown to have a role in the treatment obsessivecompulsive disorder. ${ }^{20}$ MDMA and similar drugs were originally used in psychotherapy as aids to break down anger and hostility in couples counseling. Yet once young people in the 1960s and 1970s started to use it recreationally, the public outcry led to broad reductions in availability through policy change-to the point that these medications are now unavailable even for psychiatric uses. Although MDMA is still controlled, a few medical studies have re-started particularly in the field of treatment resistant post-traumatic stress disorder (PTSD) where remarkable efficacy has been found..$^{21}$ Despite important clinical potential the illegal status of these drugs means that research has been very limited particularly in terms of using modern neuroscience techniques to unravel their actions. ${ }^{22}$ Where these barriers have been surmounted fascinating novel neuroscience data that talk to the therapeutic potential have recently been demonstrated. ${ }^{23,24}$ 
Yet, this situation is changing and the science-policy gap is narrowing, albeit slowly. This is in part because of the expanding clinical and neurosciences of addiction; adding insights into how an "addictive drug" can produce profound changes in the reward neurotransmitters and circuitry; and likely genetic expression. Neuroscience will soon inform us on how the initial drug-induced changes in neuro-circuits are then compounded as genes and emotional/behavioral processes are affected following repeated use. Ultimately this set of processes leads to initiation of negative physiological and emotional states upon cessation of use-ultimately culminating in reduced control of that drug use as discussed above.

Four points derive from this very brief, descriptive review of very complex neuroscience; and they may be helpful in creating effective clinical interventions and public policies in this area:

1. Expanding prevention and early interventions to halt emerging use seems wise based upon the available neuroscience of drug use. This research has documented that initial drug use may lead to progressive changes in neuro-circuits, producing emotional, cognitive and inhibitory reactions that shape behaviors such as drug seeking and compulsive use. The exact circumstances that lead to these changes, and the time course of the changes are still largely unknown. Regardless, it makes sense that early detection or emerging substance use followed by rapid, efficient and effective interventions may offer the best chance of averting the cascade of potentially long standing neurological, genetic, emotional and behavioral changes associated with addiction. As repeated drug use progressively alters and complicates the functions of neurotransmitter release, neuro-circuit interactions and gene expression, "abuse" becomes "addiction" and with those changes, it becomes much more difficult to regain physiological and behavioral balance.

2. Treatments for "addiction" will likely have to be chronic in nature. Given the profound and complex neurological, physiological, emotional and behavioral problems associated with engrained addiction, acute careoriented treatments involving merely education and therapy will lack sufficient potency to restore neurological balance and forestall relapses. Treatments for alcohol and other drug addictions have been traditionally short-term efforts to motivate and educate patients. The neuroscience to date offers us insights into why such short-term treatments have not produced sustained positive change. ${ }^{25-27}$ But we await more advances in clinical, pharmacological and neuroscience to produce more potent and longer acting forms of treatment that better fit what we know about the course of addiction as a chronic illness. 
3. Medications can be appropriate for treating drug addiction. When addiction is considered willfully poor self-control, it is not reasonableand perhaps even irresponsible - to think that medications might be helpful in producing recovery. Yet, the substantial body of neuroscience research shows profound and protracted physiological changes in brain systems following addiction, suggests both clear rationale and also some important specific opportunities to create effective medications that can improve behavioral and social rehabilitation efforts. In practice it can work. For example relapse to heroin can be essentially eliminated when a recovering patient has taken the opioid receptor antagonist naltrexone. Naltrexone blocks the effects of self-administered heroin or other opioids at the opioid receptor level. Intriguingly there is growing evidence opioid receptor antagonists such as naltrexone and nalmefene can also be effective in the treatment of alcohol addiction. These medications are taken before drinkers consume and when they feel that they might lose control and go on a binge. Clinical trials reveal about 30 percent fewer days of heavy drinking per month among those taking these opioid antagonists than among those in the placebo group. ${ }^{28}$ Regardless, the idea of using a drug to help control or regulate drinking is a challenging one for many therapists who have been trained to believe that abstinence through development of personal recovery and social supports is the only solution.

4. Punitive government policies have very limited ability to reduce engrained "addiction." For decades governments correctly concerned about the dangers of drug use and addiction-but uninformed about the neuroscience associated with these dangers - have enacted punishments (incarceration) and often harsh policies (permanent forfeiture of voting rights and rights to public benefits) that were designed to "teach addicts a lesson" and to "make them think twice about resuming drug use." Neuroscience has shown us that once there have been significant and pervasive changes in neuro-circuitry and perhaps genetic expression, punishing drug use among addicted individuals will likely be no more effective than punishing sugar use among diabetic patients.

\section{FUTURE OPPORTUNITIES AND CHALLENGES}

Neuroscience has produced significant advances in our understanding about fundamental processes of substance addiction. These research findings improve our insights into how the brain works and how treatments can effect change. Indeed it can be argued that addiction is among the best 
understood of the psychiatric disorders given the available knowledge from PET and SPECT imaging studies as well as "knock out" genetic studies about what drugs of abuse do to neural systems.

Despite these significant advances in our understanding about disease process, there remain significant gaps in our ability to translate neuroscience findings into better treatments and public policies. We still know too little about the interactive effects of drugs on the developing adolescent brain, or how social and emotional experiences interact with the direct effects of drugs to increase or decrease likelihood of continued use-but research has been initiated. In summary, the advances in neuroscience mean that the addiction potential of existing and new "designer" drugs can now be assessed-offering the potential for the design of more effective prevention and early interventions; and more sensible and sensitive public policies to reduce the risks and harms of drug abuse.

Conflicts of Interest: Dr. Nutt has received research support, consulting fees and speakers honoraria from Lundbeck and speakers honoraria from Reckitt Benkiser and D\&A Pharma.

\section{REFERENCES}

1. Volkow ND, Fowler JS, Wang GJ, Baler R, Telang F. Imaging dopamine's role in drug abuse and addiction. Neuropharmacology. 2009;56:3-8.

2. Di Chiara G, Imperato A. Drugs abused by humans preferentially increase synaptic dopamine concentrations in the mesolimbic system of freely moving rats. Proc Natl Acad Sci U S A. 1988;85:5274-78.

3. Nestler EJ. Is there a common molecular pathway for addiction? Nat Neurosci. 2005;8:1445-9.

4. Laruelle M. Imaging synaptic neurotransmission with in vivo binding competition techniques: a critical review. J Cereb Blood Flow Metab. 2000;20:423-51.

5. Watson BJ, Taylor LG, Reid AG, Wilson SJ, Stokes PR, et al. Investigating expectation and reward in human opioid addiction with [11C]raclopride PET. Addict Biol. 5 July 2013; epub ahead of print.

6. Nutt DJ, Lingford-Hughes A. Addiction: the clinical interface. Br J Pharmacol. 2008;154:397-405.

7. Thanos PK, Volkow ND, Freimuth P, Umegaki H, Ikari H, Roth G, et al. Overexpression of dopamine $\mathrm{D} 2$ receptors reduces alcohol self-administration. J Neurochem. 2001;78:1094-103. doi:10.

8. Volkow ND, Wang GJ, Fowler JS, Tomasi D, Telang F. Addiction: beyond dopamine reward circuitry. Proc Natl Acad Sci U S A. 2011;108:15037-42.

9. Koob GF, Volkow ND. Neurocircuitry of addiction. Neuropsychopharmacology. 2009;35:217-38. 
10. Dalley JW, Fryer TD, Brichard L, Robinson ES, Theobald DE, et al. Nucleus accumbens $\mathrm{D} 2 / 3$ receptors predict trait impulsivity and cocaine reinforcement. Science. 2007;315:1267-70.

11. Volkow ND, Wang G-J, Fowler JS, Logan J, Gatley SJ, et al. Prediction of reinforcing responses to psychostimulants in humans by brain dopamine D2 receptor levels. Am J Psychiatry. 1999;156:1440-43.

12. Martinez D, Saccone PA, Liu F, Slifstein M, Orlowska D, et al. Deficits in dopamine $\mathrm{D}(2)$ receptors and presynaptic dopamine in heroin dependence: commonalities and differences with other types of addiction. Biol Psychiatry. 2012;71:192-8.

13. Volkow ND, Wang GJ, Begleiter H, Porjesz B, Fowler JS, et al. High levels of dopamine D2 receptors in unaffected members of alcoholic families: possible protective factors. Arch Gen Psychiatry. 2006;63:999-1008.

14. Reid AG, Daglish MR, Kempton MJ, Williams TM, Watson B, et al. Reduced thalamic grey matter volume in opioid dependence is influenced by degree of alcohol use: a voxel-based morphometry study. J Psychopharmacol. 2008;22: 7-10.

15. Volkow ND, Wang GJ, Fowler JS, Logan J, Gatley SJ, et al. Decreased striatal dopaminergic responsiveness in detoxified cocaine-dependent subjects. Nature. 1997;386:830-3.

16. Nutt DJ, King LA, Phillips LD. Drug harms in the UK: a multicriteria decision analysis. Lancet. 2010;376:1558-65.

17. Weissenborn R, Nutt DJ. Popular intoxicants: what lessons can be learned from the last 40 years of alcohol and cannabis regulation? J Psychopharmacol. 2012;26:213-20.

18. Carter LP, Stitzer ML, Henningfield JE, O’Connor RJ, Cummings KM, Hatsukami DK. Abuse liability assessment of tobacco products including potential reduced exposure products. Cancer Epidemiol Biomarkers Prev. 2009;18:3241-62.

19. Krebs TS. Johansen PØ. Lysergic acid diethylamide (LSD) for alcoholism: meta-analysis of randomized controlled trials. J Psychopharmacol. 2012;26: 994-1002.

20. Moreno FA, Wiegand CB, Taitano EK, Delgado PL. Safety, tolerability and efficacy of psilocybin in 9 patients with obsessive-compulsive disorder. J Clin. Psychiatry. 2006;67:1735-40.

21. Mithoefer MC, Wagner MT, Mithoefer AT, Jerome L, Doblin R. The safety and efficacy of $\pm 3,4$-methylenedioxymethamphetamine-assisted psychotherapy in subjects with chronic, treatment-resistant posttraumatic stress disorder: the first randomized controlled pilot study. J Psychopharmacol. 2010;25:439-52.

22. Nutt DJ, King LA, Nichols DE Effects of Schedule1 drug laws on neuroscience research and treatment innovation. Nature Rev Neurosci. 2013;14:577-85. 
23. Carhart-Harris RL, Murphy K, Leech R, Erritzoe D, Wall MB, et al. The effects of acutely administered 3, 4-methylenedioxymethamphetamine on spontaneous brain function in healthy volunteers measured with arterial spin labelling and blood oxygen level-dependent resting-state functional connectivity. Biol Psychiatry. 2014; epub ahead of print.

24. Carhart-Harris RL, Leech R, Hellyer PJ, Shanahan M, Feilding A, et al. The entropic brain: A theory of conscious states informed by neuroimaging research with psychedelic drugs. Front Hum Neurosci. 2014;8:20.

25. McLellan AT, Lewis DC, O'Brien CP, Kleber HD. Drug dependence, a chronic medical illness. JAMA. 2000;284:1689-95.

26. McLellan AT, McKay JR, Forman R, Cacciola J, Kemp J. Reconsidering the evaluation of addiction treatment: from retrospective follow-up to concurrent recovery monitoring. Addiction. 2005;100:447-58.

27. McLellan AT, Skipper GS, Campbell M, DuPont RL. Five year outcomes in a cohort study of physicians treated for substance use disorders in the United States. BMJ. 2008;337:a2038.

28. Mann K, Bladström A, Torup L, Gual A, van den Brink W. Extending the treatment options in alcohol dependence: a randomized controlled study of as-needed nalmefene. Biol Psychiatry. 2013;73:706-13.

29. Volkow ND, Wang GJ, Fowler JS, Tomasi, D. Addiction circuitry in the human brain. Annu Rev Pharmacol Toxicol. 2012;52:321-36. 